\title{
A Master Shape of Bottles for Design under Desirable Geometry and Top Load Test
}

\author{
Piyapat Keawjaroen and Chakrit Suvanjumrat \\ Department of Mechanical Engineering, Faculty of Engineering, Mahidol University, Salaya, Nakorn Pathom, 73170, Thailand \\ Laboratory of Computer Mechanics for Design (LCMD), Department of Mechanical Engineering, Faculty of Engineering, Mahidol University, \\ Salaya, Nakorn Pathom, 73170, Thailand
}

\begin{abstract}
Design of plastic bottles had preferred to use computer aided design (CAD) to propose desirable shapes. The strength also was regarded to pass the top load test unless an appearance of plastic bottles. Finite element method (FEM) was employed to analyze and predict the bottle shape which enough to support load under a collapsible regulation. Unfortunately, the redesign of bottle shape always performed when the desirable bottle shape had not passed the test. There was time consumption and loss of opportunity to compete producing of bottles. This research proposed a method to receive a desirable shape of plastic bottles together with top load strength. The master of bottle shape had been created which capable to change any dimensions before generated CAD and performed top load analysis with FEM. The artificial neural network (ANN) was employed to obtain the desirable bottle shape with top load resistance by varying dimension of the master bottle. The plastic bottle design would be performed rapidly with the ANN of master bottle shape. Consequently, the suitable dimension of plastic bottles which achieved by ANN could be used to design a desirable shape of bottles by using CAD and FEM without trial and error.
\end{abstract}

\section{Introduction}

Plastic bottles were produced to contain liquid must be had strength to support top load causes a warehousing according to ASTM D2659 [1]. To reduce trial and error by production, computer aided design (CAD) and computer aided engineering (CAE) had been used to design and analyze bottle shape before real production [2]-[4]. Unfortunately, the bottle production will use $\mathrm{CAD} / \mathrm{CAE}$ method repeatedly until achieves a desirable shape of plastic bottles. The product cost will be increased by a consumption of time and design number. Furthermore, the opportunity to achieve production of plastic bottles from customer will be lost.

Artificial neural network (ANN) is a computational model that mimics human brains to learn relation between inputs and outputs from experiment. Many researchers have applied ANN to design products [5]-[7]. The important feature of ANN is the ability to estimate complex relationships without any prior assumptions about the nature and interrelations of the underlying process. The transfer functions, weight and output are constructed the neural network to recieve input and predict output, respectively [8]. The ANN is trained by input and output data until it can predict output by itsilf [9] [10].

This research has developed the design process of plastic bottles by using ANN. The master shape of 1 liter bottle has created and analyzed the top load resistance.
The simulation data is used to train ANN which is the design tool of plastic bottle. The desirable shape of plastic bottle which achieve by ANN will be created CAD model and analyzed with FEM of top load test to produce plastic bottles in the future.

\section{Master shape of bottle}

\subsection{CAD model}

The bottle model is created by classify a capacity of 1 liter, no handle and rectangular base from all bottle products of the company. The master bottle shape is defined and generated by SolidWorks software as illustrated by in Fig. 1. The shape of bottle can vary by curvatures which include $\mathrm{R}_{1}, \mathrm{R}_{2}, \mathrm{R}_{3}, \mathrm{R}_{4}$ and $\mathrm{R}_{5}$. The curvature of bottle neck $\left(\mathrm{R}_{5}\right)$ has many effects to collapse bottles by top load test therefore it is extreamely brought to be a parameter of the master shape. The bottle width is limitted to be constant with a value of $109.51 \mathrm{~mm}$. Fig. 1 shows the 3D-model of the master shape bottle. The bottle neck does not have a screw thread detail because it does not have any effect to simulate the top load test for the bottle shape design. The diameter of bottle neck is limited at $36.55 \mathrm{~mm}$ while the height is $232.42 \mathrm{~mm}$. The curvatures of the master model have relation to the bottle geometry therefore it has a dimensional limitation for creating of the 1 liter bottle model to produce the real bottle shape in manufacturing process. 


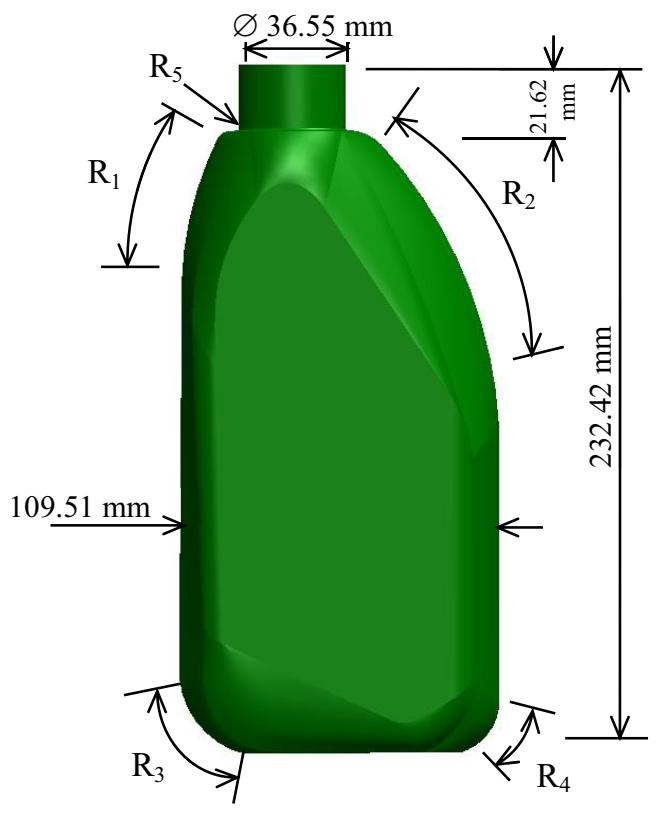

Figure 1. The variable dimension of a 1 liter master shape bottle.

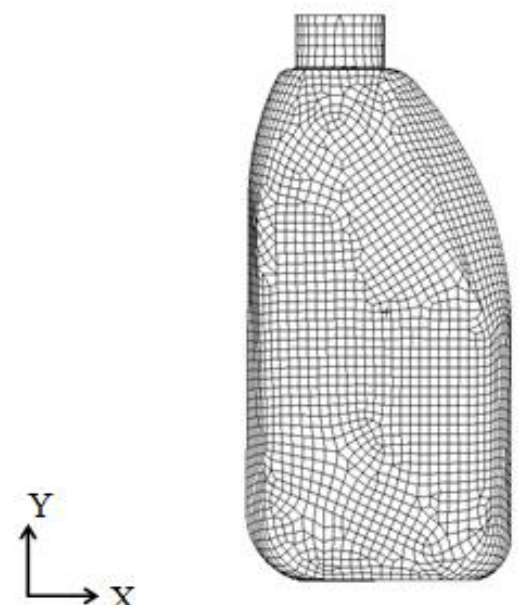

(a)
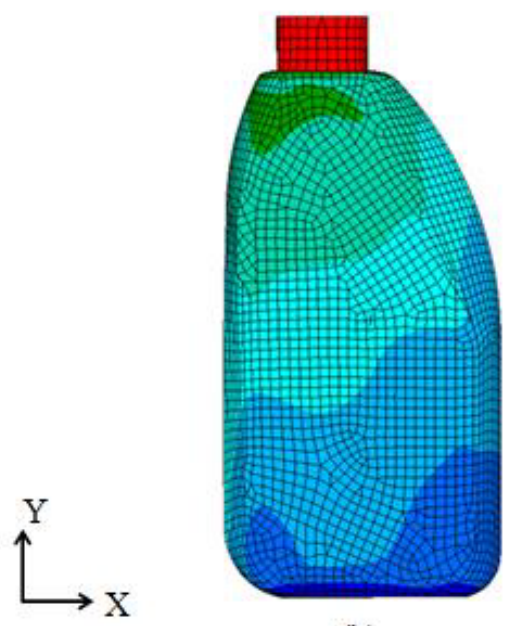

Unit: $\mathrm{mm}$

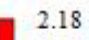

2.04

1.89

1.75

1.60

1.45

1.31

1.16

1.02

0.87

0.73

0.58

0.44

0.29

0.14

(b)

Figure 2. The finite element model of the master shape bottle under top load analysis as a result of: (a) a mesh structure and (b) mesh deformation with a color contour of the vertical displacement.

\subsection{Top load analysis}

The CAD model of a master shape is transformed into finite element analysis (FEA) software, MSC.Nastran, to simulate top load test with a constant mass of $25 \mathrm{~kg}$. The quadrilateral plate element is used to create the finite element model of the master shape bottle as shown in Fig. 2a. The total plate elements to created finite element model are 4,625 elements. The thickness of plate elements or the bottle wall is assign to be a constant value which is $1 \mathrm{~mm}$. The 1 liter bottle is produced by the high density polyethylene (HDPE) material by the extrusion blow molding process. Therefore, the material properties of the bottle model obtain by the tensile test of HDPE specimens according to ASTM D638 [11]. The Young's modulus and a yield stress of the HDPE material is $522.45 \mathrm{MPa}$ and $27.34 \mathrm{MPa}$, respectively. The bottle model has been supported the vertical top load on the bottle neck therefore it is collapsed. The deformation of bottle wall is illustrated by color contour which red is the maximum vertical displacement and dark blue is the minimum vertical displacement (Fig. 2b). The maximum vertical displacement of the bottle deformation which varies by the variable shape of the master shape bottle is used to be the output data while curvatures of the master bottle is the input data of ANN, respectively.

\section{Artificial neural networks}

The architecture of ANN in this research is using the error back-propagation neural network model. The multiple-input neuron includes of the 5 neural units. The multiple-hidden layer includes of 20 tansig, 10 tansig and 5 logsig functions. The output is the vertical deformation of plastic bottles by the top load simulation teat which is one neural unit. The five-layered supervised ANN for this research is described by a diagram as shown in Fig. 3 . Each neuron operates by the sum of weighted inputs and passes the result through a transfer function of network which is modeled as:

$$
\begin{aligned}
o_{p k}=f_{k}\left(\text { net }_{p k}\right)= & f_{k}\left(\sum _ { n } W _ { k n } f _ { j } \left(\sum _ { m } W _ { j m } f _ { i } \left(\sum_{l} W_{i l} O_{i l}\right.\right.\right. \\
& \left.\left.\left.+\theta_{i}\right)+\theta_{j}\right)+\theta_{k}\right)^{-}
\end{aligned}
$$

where $f_{k}\left(n e t_{p k}\right)$ is a transfer function in $k$-layer, $O_{i l}$ is the output in $i$-layer, $\theta_{i}$ is the threshold in $i$-layer, $\theta_{j}$ is the threshold in $j$-layer, $\theta_{k}$ is the threshold in $k$-layer, $W_{i l}$ is weight of connection in $i$-layer, $W_{j m}$ is weight of connection in $j$-layer, $W_{k n}$ is weight of connection in $k$ layer, $O_{p k}$ is the output in the current layer.

The tansig and logsig transfer function is written by:

$$
\begin{aligned}
O_{p i} & =\frac{e^{\left(n e t_{p i}\right)}-e^{-\left(\text {net }_{p i}\right)}}{e^{\left(\text {net }_{p i}\right)}+e^{-\left(\text {net }_{p i}\right)}} \\
O_{p k} & =\frac{1}{1+e^{-\left(\text {net }_{p k}\right)}}
\end{aligned}
$$




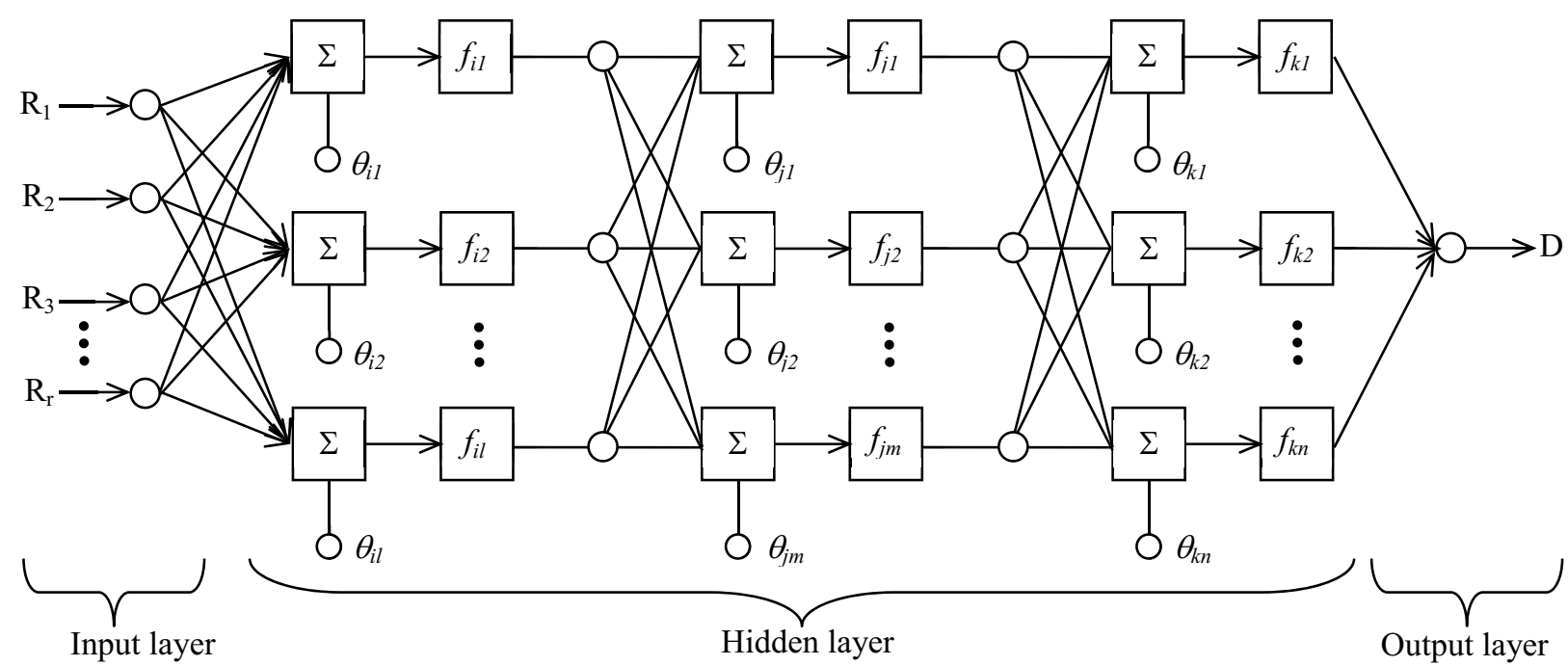

Figure 3. Structure of five-layer ANN.

where $O_{p k}$ is the output of network.

The iterative procedure to adjust weights in each step uses the Levenberg-Marquardt algorithm which is given by:

$$
W_{i l}(n+1)=W_{i l}(n)-e_{i l} \frac{J_{n}^{T}}{\left(J_{n}^{T} J_{n}+\mu_{n} I\right)}
$$

where

$$
\begin{gathered}
J=\left[\frac{\partial e_{i l}}{\partial W_{i l}}\right] \\
e_{i l}=\left[\begin{array}{c}
T_{i 1}-Q_{i 1} \\
T_{i 2}-Q_{i 2} \\
T_{i 3}-Q_{i 3} \\
\vdots \\
T_{i l}-Q_{i l}
\end{array}\right]
\end{gathered}
$$

where $T_{i l}$ is the target output of $i$-layer, $J$ is the Jacobian matrix which includes the first order partial derivatives of the network errors with respect to weights, $e_{i l}$ is an error vector of $i$-layer corresponding to the $l$-data, $I$ is the identity matrix. $\mu_{n}$ is constant between 0 and 1 .

\section{Results and discussion}

The 1 liter bottle was design depending on the desirable geometry by using the master shape bottle. The curvatures of desirable bottle products included $\mathrm{R}_{1}, \mathrm{R}_{2}$, $R_{3} R_{4}$ and $R_{5}$ are selected following of 140.00, 240.00, $30.00,20.00$ and $0.97 \mathrm{~mm}$, respectively. The desirable bottle was not decorated by ribs or grooves on its wall until there were certain for the corrected shape. Fig. 4 shows model of the bottle which will be predicted the vertical deformation of top load test by ANN. This bottle model achieved the maximum vertical deformation of $2.08 \mathrm{~mm}$ which would pass the standard test of the company (less than $4.00 \mathrm{~mm}$ ).

The bottle model was analyzed the top load test by FEM. The quadrilateral plate element was used to create the finite element model of the desirable bottle. The total plate elements were 4,552 elements. The bottle wall was
HDPE material which was assigned a constant thickness of $1 \mathrm{~mm}$. The bottle model had been supported the vertical top load on the bottle neck at $25 \mathrm{~kg}$. The FEA results of the top load test on the desirable shape bottle show in Fig. 5. The maximum vertical deformation of the 1 liter bottle model which analyzed by FEA was $2.09 \mathrm{~mm}$. The FEA results expressed the vertical deformation of the desirable shape bottle less than the company's standard of $1.91 \mathrm{~mm}$ therefore it certainly passed the top load test.

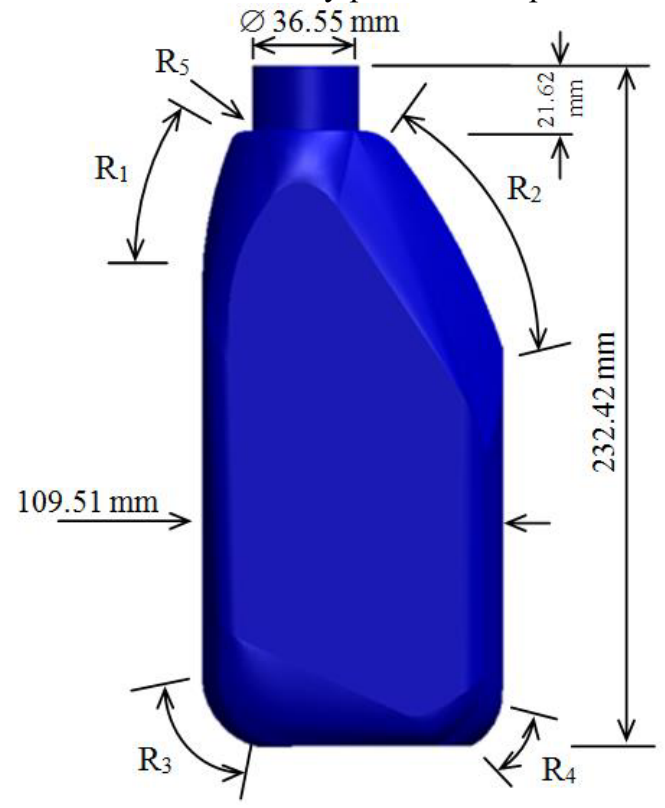

Figure 4. The CAD model of the desirable shape bottle.

The final bottle shape was decorated by groove and sticker area and then it brought to create mold for producing by the extrusion blow molding process. The real 1 liter bottle has been test according to the ASTM D2659 using the universal testing machine (Hounsfield: model H50KS) as shown in Fig. 6. The bottle samples were pressed down on it's neck in the vertical direction until collapsing. Every bottle first collapsed at neck round $\left(R_{5}\right)$ and moved to bottom round $R_{4}$ and $R_{3}$, respectively. The resistant force was equal to the top load which was recorded by a load cell of the universal testing machine 
during sample tests. Five bottles give testing results which show by relation graph between top load and vertical deformation in Fig. 7. The average deformation of the bottle test expressed the vertical deformation by the top load at $250 \mathrm{~N}(25 \mathrm{~kg})$ was $2.40 \mathrm{~mm}$. The vertical deformation of the ANN was different from the FEA and

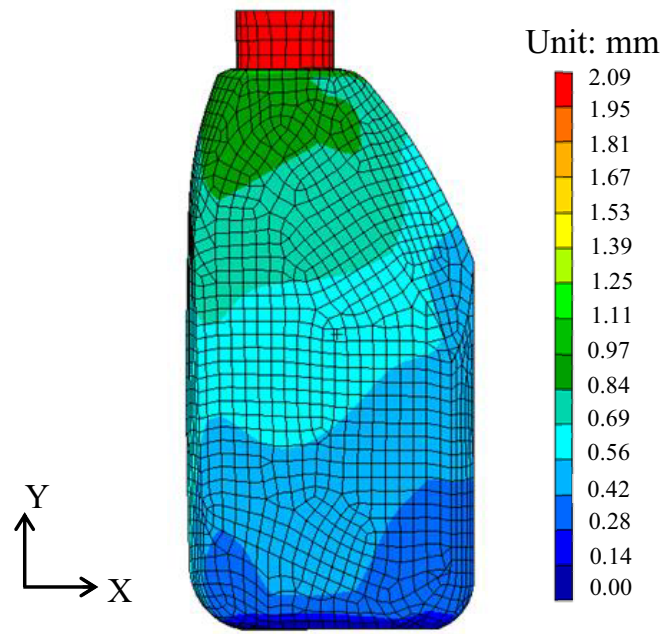

Figure 5. The FEA results of the top load testing under the constant weight of $25 \mathrm{~kg}$.

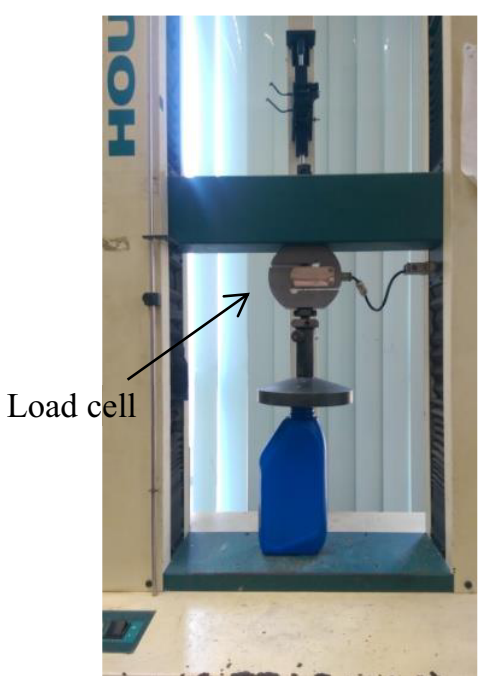

Figure 6. The top load test of the 1 liter bottle using universal testing machine.

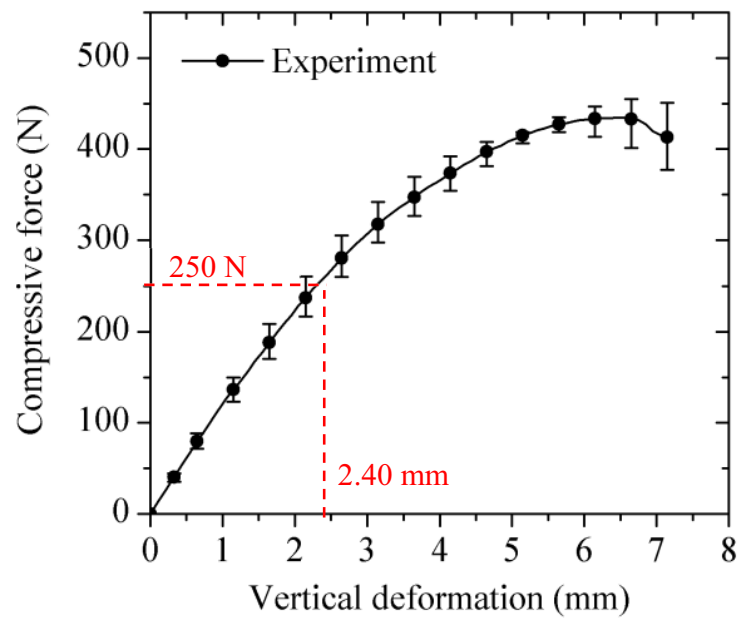

Figure 7. The top load test result of the 1 liter bottle. the physical experiment for the top load test was -0.02 and $-0.32 \mathrm{~mm}$, respectively.

\section{Conclusions}

The master shape of the 1 liter bottle which had the rectangular base and capability to adjust geometry had been created to use for the bottle design. The ANN training had performed to predict the vertical deformation of bottle models under the top load test at $25 \mathrm{~kg}$. The master shape of bottle could used to design shape to pass the top load test by using ANN at the vertical deformation of company specification. Furthermore, the desirable bottle model had been reexamined by FEA before brought to produce. The bottle product had been test by the universal testing machine again and was found the different deformation from the ANN and FEA. The ANN obtained an error less than $-13.33 \%$ when compared with the experimental data. Nevertheless, the ANN result passed the top load test. Finally, the ANN training of the master shape bottle under the top load test can be used to be the design tool for the 1 liter HDPE bottle of the company in the future.

\section{Acknowledgement}

This research was funded by the Thailand Research Fund (TRF) and Panjawattana Plastic Public Company Limited under Research and Researchers for Industries (RRI): Master Degree-grant no. MSD58I0051.

\section{References}

1. ASTM International, D2659-11 standard test methods for column crush properties of blown thermoplastic containers, ASTM International, PA, (2011)

2. C. Suvanjumrat, E. Chaichanasiri, Kasetsart J. (Nat. Sci.) 48, 3 (2014)

3. C. Suvanjumrat, T. Puttapitukporn, Kasetsart J. (Nat. Sci.) 45, 5 (2011)

4. C. Suvanjumrat, T. Puttapitukporn, S. Thusneyapan, Kasetsart J. (Nat. Sci.) 42, 1 (2008)

5. H. Avci, D. Kumlutas, O. Ozer, M. Ozsen, Int. J. Refrig. 67 (2016)

6. B. Wimarshana, J. Ryu, H.J. Choi, Int. J. Precis. Eng. Man. 15, 1 (2014)

7. C.C. Zhou, G.F. Yin, X.B. Hu, Mater. Design 30 (2009)

8. M.F. Augusteijn, T.P. Harrington, Neural Compt. \& Applic. 13 (2004).

9. D.J. Livingstone, Artificial Neural Networks: Methods and Applications, Humana Press, UK, (2009)

10. S. Rajasekaran, G.A. Vijayalakshmi Pai, Neural Networks, Fuzzy Logic, and Genetic Algorithms: Synthesis and Applications, PHI, New Delhi, (2008)

11. ASTM International, D638-14 standard test methods for tensile properties of plastics (ASTM International, PA, 2014) 